\title{
DE LA ÉTICA AL DERECHO EN LA OBRA DE ALASDAIR MACINTYRE *
}

\author{
Rafael Ramis Barceló \\ Universitat de les Illes Balears
}

RESUMEN. En este artículo se compendian las ideas principales de A. MACINTYRE sobre el derecho natural. El trabajo empieza con una visión general de las ideas políticas y éticas de MACINTYRE. Luego se define su posición en el debate con los filósofos del derecho actuales, para pasar luego a su postura en el debate sobre el derecho natural, subrayando algunas dificultades en su concepción. El artículo concluye considerando cómo las ideas de MACINTYRE pueden arrojar luz para solucionar los problemas actuales.

Palabras clave: MACINTYRE, derecho natural, ética, liberalismo, razones para la acción.

ABSTRACT. In this article summarizes the main ideas of Alasdair MACINTYRE on Natural Law. The paper begins with an overview of ethical and political ideas dealt with by MACINTYRE. Then it is defined his position in the debate between current legal philosophers before moving on to his position on debate on Natural Law and highlighting some difficulties in his conception. The paper closes by focusing on how many of MACINTYRE's ideas can shed light on solutions to current problems.

Keywords: MACINTYRE, natural law, ethics, liberalism, reasons for action.

* Fecha de recepción: 15 de agosto de 2012. Fecha de aceptación: 15 de octubre de 2012. 
n este escrito quisiera compendiar algunas de las principales conclusiones de mis estudios sobre el pensamiento jurídico de A. MACINTYRE (Glasgow, 1929), un autor que no ha dedicado ningún libro exclusivamente a cuestiones jurídicas. He espigado en su obra prácticamente todos aquellos puntos que hacen referencia al derecho (y que se encuentran diferentes apartados de libros, artículos o recensiones) y he intentado una visión unitaria ${ }^{1}$, que intento explicar en las páginas siguientes.

Empiezo con una exposición general de las ideas ético-políticas de MACINTYRE para intentar delimitar luego su posición en el debate iusfilosófico actual. Acabo mi escrito con una serie de comentarios que muestran las dificultades para articular, a partir de los escritos del profesor escocés, una filosofía del derecho en general y, en concreto, la defensa de una doctrina iusnaturalista ${ }^{2}$. Con todo, en la obra del escocés pueden encontrarse muchas ideas capaces de iluminar problemas actuales.

\section{RAZONES PARA ACTUAR Y LIBERALISMO}

En los años cincuenta y en los sesenta, MACINTYRE está muy interesado en el divorcio entre razón teórica y razón práctica. Dicha brecha resulta muy problemática para articular «razones para actuar» ${ }^{3}$. Su posición ya entonces está muy cerca de ARISTÓTELES y del segundo WITTGENSTEIN y sus autores de cabecera son seguidores de uno u otro, o bien de ambos (cabe mentar a ANSCOMBE, VON WRIGHT y HART).

En los años cincuenta y sesenta, MACINTYRE anda buscando también una respuesta a su modelo explicativo en las obras de MARX y de HEGEL ${ }^{4}$. Marx elabora una interpretación de las razones como un compromiso político, una vinculación con la praxis. HEGEL está interesado en explicar la acción como el desarrollo histórico del espíritu y muestra que hay ciertas diferencias entre antiguos y modernos ${ }^{5}$. MACINTYRE se percata en aquel momento de que, ciertamente, los griegos tenían razones para actuar y los modernos carecían de ellas. Su conclusión es que algo se había perdido en el viaje dialéctico del espíritu. MACINTYRE entiende que la Ilustración y la pujanza de las ciencias naturales habían reducido la explicación de la acción a un modelo conductista, basado exclusivamente en las causas, que no era válido para las ciencias sociales.

\footnotetext{
1 Una visión más extensa puede verse en R. RAMIS BARCELÓ, Derecho natural, historia y razones para actuar. La contribución del A. MacIntyre al pensamiento jurídico, Madrid, Carlos III-Dykinson, 2012.

2 Vid. P. Riordan, «Natural Law Revivals: A Review of Recent Literature», The Heythrop Journal, 51, 2010, 314-323. Vid. también M. MAURI, «Alasdair MacIntyre: On Natural Law» en A. N. GARCÍA MARTÍNEZ, M. Silar y J. M. Torralba (eds.), Natural Law: Historical, Systematic and Juridical Approaches, Cambridge, Cambridge Scholars Publishing, 2008, 259-266, y S. MALETTA, «MacIntyre and the Subversion of Natural Law», en P. Blackledge y K. Knight (eds.), Virtue and Politics, Notre Dame, University of Notre Dame, 2011, 177-194.

3 Vid. P. Blackledge y N. Davidson (comps.), A. MacIntyre's Early Marxist Writings: Essays and Articles 1953-1974, Brill, Leiden, 2008.

4 Vid. R. RAMIS BARCELÓ, «MacIntyre y el marxismo: Historia, compromiso y razones para la acción», Crítica. Revista Hispanoamericana de Filosofía, vol. 43, núm. 127, 2011, 77-87.

5 A. MacInTyre, A Short History of Ethics, New York, Macmillan, 1966, 199.
} 
Como es sabido, WiTTGENSTEIN distingue entre causas y razones y MACINTYRE, siguiéndole a él y a su discípula ANSCOMBE, considera que para explicar la acción es necesario un proceso de deliberación ${ }^{6}$. La concepción fenomenológica ayuda a superar el dualismo de los científicos y de los positivistas que separan la acción de la historia y creen que la ciencia sólo se limita a la observación y a la descripción. MACINTYRE, al contrario, siguiendo al segundo WiTTGENSTEIN, cree que la acción está ejecutada (y descrita) por un sujeto, al igual que las prácticas socialmente constituidas no pueden ser separadas del contexto en las que se producen.

Para MAcInTyre, desde su obra A Short History of Ethics (1966) el problema de las razones para la acción exige una aproximación histórica y lingüística. MACINTYRE busca estudiar el lenguaje de la ética a través de los diferentes periodos ${ }^{7}$ y su análisis demuestra que hay una brecha entre antiguos y modernos que ni el conductismo ni el compromiso marxista pueden explicar. A partir de A Short History of Ethics, MAcINTYRE sabe que existe una diferencia histórica en el lenguaje de la ética, pero carece de una teoría adecuada para explicarla ${ }^{8}$.

La pérdida de la narrativa en la historia puede verse, según MACINTYRE, en la pobreza del lenguaje de la política, de la moral y de la justicia en la Modernidad ${ }^{9}$. El contraste entre antiguos y modernos muestra la falta de contexto del léxico moderno y que deber y acción están separados de la Historia. El análisis del lenguaje ético y político revela que el liberalismo disocia de las razones para la acción de su contexto. A partir de las obras de HOBBES y KANT, la atomización moral y política del individuo provoca una pérdida del sentido de comunidad ${ }^{10}$.

La pérdida del contexto lingüístico en una comunidad lleva a MACINTYRE a defender una interpretación histórico-hermenéutica a partir del concepto de tradición, de manera que las razones para actuar pasen a entenderse en clave hermenéutica. El escocés cree que para entender las acciones de las personas es necesario empezar con el análisis del sistema de creencias en las que se refleja el lenguaje compartido. Las razones para la acción toman sentido sólo bajo un conjunto de referencias morales que no son «razones éticas» de aplicación universal, sino de uso particular o grupal.

Para MacInTYRe, las razones para la acción empiezan primero como deseos e intenciones del agente (las cuales pueden ser explicadas sólo en el contexto de las creencias compartidas) y luego, a partir de la aplicación del silogismo práctico de ARISTÓTELES (que comparte también con VON WRIGHT), se llega a la conclusión de que el hombre debe actuar. Con ello, MACINTYRE intenta vincular las ideas de WITTGENSTEIN (reglas sociales) con la fenomenología y la razón práctica de ARISTÓTELES.

MACINTYRE cree que el liberalismo está equivocado al considerar que el hombre es esencialmente autónomo y que su acción está separada de su conocimiento. La ciencia,

6 A. MACINTYRE, «The antecedents of action», en Against the Self-Images of the Age, Essays on Ideology and Philosophy, London, Duckworth, 1971, 207-210.

7 A. MacInTYRe, A Short History of Ethics, op. cit., 1-12.

8 Ibid., 265.

9 A. MAcInTYRe, «Justice: A New Theory and Some Old Questions», Boston University Law Review, 52, 1972,330-4.

10 A. MacInTYRe, «On Democratic Theory: Essays in Retrieval by C. B. MacPherson», Canadian Journal of Philosophy, 6, 1976, 180. 
en el entramado conceptual del liberalismo, considera que las razones y las intenciones del agente no tienen ninguna relación con el resultado que se obtiene y que la ciencia social debería limitarse, al igual que la natural, a describir las acciones y a buscar las causas a partir de los efectos ${ }^{11}$.

En contra de esta visión, en los años setenta MACINTYRE cree haber determinado un método nuevo, siguiendo al segundo WitTGENSTEIN. Considera que las razones para actuar no son mecánicas sino que el sujeto actúa racionalmente si es capaz de narrar (explicar) sus propias deliberaciones. La explicación toma sentido sólo en el contexto en el que un grupo de hablantes puede entenderse.

Por el contrario, las ciencias sociales —en las últimas décadas— más que buscar una narrativa, intentan conformarse con la asepsia del liberalismo ${ }^{12}$. Para MACINTYRE, la teoría liberal aprueba e incluso contribuye a que las ciencias sociales (y la historia en particular) no hallen una jerarquía causal. La ideología liberal-burocrática, según el escocés, se alía con la ciencia para deshumanizar la explicación histórica y social. El liberalismo, aliado con el reduccionismo de la ciencia moderna, permite en su ahistoricismo que toda obligación se tome como un simple imperativo ${ }^{13}$.

MACINTYRE propone una visión de las razones para la acción de acuerdo con la ética griega, vinculando las acciones con las intenciones y los propósitos, de manera que se vincule también la historia y su contexto. El autor sostiene que la razón es lo que permite una explicación causal de la historia: así, la teleología de carácter aristotélico es capaz de dar sentido y explicación a la historia, incluyendo también los cambios y las revoluciones en el seno de una misma tradición ${ }^{14}$.

Sólo a través de una racionalidad falsable y narrativa pueden explicarse las razones para la acción como parte de una comunidad que da relevancia al intento, al deseo o a la deliberación. A falta de una narrativa racional, de acuerdo con MACINTYRE, sólo existen imperativos, acciones egoístas y una proliferación de normas jurídicas sin soporte moral. Hay que incardinar el deber en una tradición narrativa y así el sujeto puede explicar (a sí mismo y a los demás) por qué actúa. Las historias tienen que ser evaluadas por otros y, para ello, tienen que ser explicadas y compartidas en términos racionales (tal y como exigen POPPER y KUHN).

Si se añaden a A Short History of Ethics algunas consideraciones sobre el lenguaje del liberalismo, la percepción fenomenológica de la tradición y de la narrativa, así como la justificación epistemológica de la metodología de la ciencia, MACINTYRE tiene todos los elementos para dar una respuesta al problema las razones para la acción en la sociedad contemporánea. Juntando las ideas anteriores, MACINTYRE concluye su explicación histórica de la actual falta de razones para la acción sosteniendo que en nuestra época vivimos una sociedad moralmente fragmentada y que sus miembros tie-

${ }_{11}$ A. MacInTyre, «Predictability and Explanation in the Social Sciences», Philosophical Exchange, 1(3), 1972, 5-13.

12 A. MAcInTyre, «The Idea of Social Science», Aristotelian Society, 41, 1967, 95-114.

13 A. MacIntyre, «Causality and History», en J. Manninen y R. Tuomela (eds.), Essays on Explanation and Understanding: Studies in the Foundations of Humanities and Social Sciences, Dordrecht and Boston, Reidel, 1976, 137-158.

${ }_{14}$ A. MacInTYRe, «Epistemological Crises, Dramatic Narrative and the Philosophy of Science», The Monist, 60, 1977, 453-72. 
nen muy poco en común con los demás. Por el contrario, la sociedad está basada en un frágil acuerdo de voluntades (que es político y no moral). Por ello, existe una auténtica proliferación de normas legales y de pleitos, pues la doctrina liberal ha favorecido una atomización tan absoluta del sujeto que, sin una continua remisión al derecho, no puede articular su convivencia ${ }^{15}$.

En la época moderna, con el ascenso del liberalismo, se pasó de una comprensión moral del derecho (que permitía la presencia de unos derechos naturales de la persona) a una comprensión política del derecho (usada para proteger instituciones, las libertades políticas y la propiedad). Para MAcInTYRE, al final del XVIII se dio una transición desde una antropología basada en la persona de carne y hueso a una antropología trascendental, basada en el sujeto trascendental. Ambos modelos (político y antropológico) implicaban la pérdida de la moral y de la antropología natural en el marco político, y dichos cambios tuvieron su manifestación en el positivismo jurídico.

La subordinación del derecho a la política permitió el ascenso del positivismo, que aspira a una justicia sólo en un sentido formal. El liberalismo, en definitiva, no entiende la justicia como una virtud ética, sino como un estándar político ${ }^{16}$. Las obras de RAWLS y DWORKIN muestran la naturaleza política de la justicia, pero sólo en una visión puramente formal. El derecho depende de unos criterios técnicos, basados en intereses políticos, y no tiene ninguna relación con la ética ni con la antropología.

Para MAcInTYRe, el liberalismo se centra en una visión formal del derecho, que sólo garantiza libertades formales, ideales, abstractas. No es capaz de resolver los desacuerdos morales porque no entra en el contenido material de los mismos ${ }^{17}$. He aquí la causa de la proliferación los desacuerdos morales irresolubles. Para MACINTYRE, la solución de los mismos debe pasar por el estudio de la antropología y de las inclinaciones morales. Así, siguiendo a ARISTÓTELES y a T. DE AQUINO ${ }^{18}$, es necesario restaurar las virtudes naturales propias para resolver dilemas y desacuerdos morales.

\section{HACIA EL DERECHO NATURAL}

MACINTYRE, a finales de los setenta y comienzos de los ochenta, cree haber resuelto el problema de las razones para actuar a través de una comprensión histórica. Para él, ARISTÓTELES es el único filósofo capaz de sobreponerse al nihilismo actual, e incluso a la crítica que hizo NIETZSCHE a la ética occidental. La obra de ARISTÓTELES sirve para unir el derecho y la acción a través de la deliberación. En su obra After Virtue, muestra que la Modernidad ha perdido la ética de la virtud y ha cultivado el formalismo liberal, dejando de lado la objetividad de la moralidad. MACINTYRE propone una vuelta a ARISTÓTELES porque la Modernidad ha tomado un camino equivocado ${ }^{19}$.

Para MacInTYRe, los desacuerdos morales existen porque no hay bases que puedan explicar el contenido material de la propia moral. La Modernidad liberal

\footnotetext{
15 A. MaCINTYRE, «Regulation: A Substitute for Morality», Hastings Center Report, 10/1 (febrero 1980), 32.

16 Ibid., 32.

17 A. MacInTYre, After Virtue, Notre Dame, University of Notre Dame Press, 1981, 86.

18 T. De Aquino, Quaestio Disp. De Veritate, q. 1, c. 17, art. 4.

19 A. MacInTYRE, After Virtue, op. cit., 118.
} 
rechaza la capacidad para entrar en el contenido material y objetivo de la moral. ARISTÓTELES da una explicación completa de la realidad, basada en la antropología, biología, psicología y sociología de los seres humanos. Mientras que ARISTÓTELES construye su ética sobre las bases de las inclinaciones naturales, necesidades, deseos e impulsos, la Modernidad defiende una doctrina sofisticada contraria a dichos impulsos.

Con todo, la explicación aristotélica tiene - para MACINTYRE- un punto débil: no tiene una fundamentación fuerte para el deber y las reglas, y falta un vínculo entre la virtud de la justicia y la imperatividad del derecho. Para ARISTÓTELES, la felicidad del hombre es la meta para sí mismo y para la humanidad; sin embargo, no hay una fuente externa que obligue a llevar a cabo la acción virtuosa y que permita conectar el deber con la virtud. La justicia y la imperatividad sólo tienen un carácter teleológico basado en la antropología y en la sociología. Carecen de una fuente externa que dote de imperatividad hacia la virtud, ya que la teleología muestra sólo la inclinación hacia un fin, pero no la obligatoriedad (deóntica) de cumplirlo.

Frente a la deontología kantiana, MACINTYRE busca refinar el sistema aristotélico con una fuente que le asegure que la finalidad teleológica desemboca en una moral objetiva y que el conocimiento del bien dé lugar a su efectiva realización. Así, el escocés entiende que T. DE AQUiNo mejora sustancialmente la obra de ARISTÓTELES porque apuntala un concepto de Dios mucho más fuerte y que existe como garante de la virtud, del bien y de la verdad, y que asegura que la virtud tenga que ser practicada y que el conocimiento de la virtud de la justicia no pueda deslindarse de un cumplimiento del derecho que está en consonancia con esa virtud y el bien.

La razón práctica conoce lo que es bueno y lo que es malo. Dios no se limita a una garantía cartesiana de la verdad, sino que Dios obliga a hacer el bien y a evitar el mal. El Dios cristiano, como muestra T. DE AQUINO, no formula nada contra las inclinaciones naturales (sociológicas y antropológicas) de ARISTÓTELES, sino que las perfecciona $^{20}$. Dios garantiza que haya una serie de bienes y de derechos que no pueden ser objeto de negociación. A diferencia de los «derechos humanos» ${ }^{21}$, que MACINTYRE entiende como una ficción, están los «derechos naturales», enraizados en la naturaleza humana. Sólo Dios puede asegurar la inclinación hacia la verdad y puede formular una prohibición clara contra determinadas actitudes para proteger bienes y derechos. Así, nunca un «derecho humano» puede prohibir, por ejemplo, de manera tan radical la esclavitud como el «derecho natural».

Este viaje al corazón de la tradición aristotélico-tomista permite descubrir la superioridad de esta tradición sobre otras, ya que permite dar una explicación completa de la realidad, integrando todas las dimensiones del ser humano. A diferencia del formalismo liberal ${ }^{22}$, que escinde al sujeto, la tradición aristotélica pone a los seres humanos en contacto con una realidad global, permitiendo un acceso al summum bonum. Para la razón es posible conocer el bien si la virtud se ejerce de acuerdo con él. La justicia

20 A. MacIntyre, «Which God Ought We to Obey and Why?», Faith and Philosophy, 3, 1986, 364 ss.

21 A. MacIntyre, Three Rival Versions of Moral Enquiry, Notre Dame, University of Notre Dame Press, 1990,76

${ }_{22}$ A. MacInTyre, Whose Justice? Which Rationality?, Notre Dame, University of Notre Dame Press, 1988, 108-109. 
es la virtud ética por excelencia que conecta lo individual con la sociedad ${ }^{23}$. Donde la doctrina liberal pone el acento sobre los derechos individuales, la tradición aristotélica se centra en el bien común.

MACINTYRE, traza un proyecto con dos ramificaciones: el primero es la discusión de las doctrinas éticas y jurídicas contemporáneas, y el segundo es la explicación de los orígenes éticos y jurídicos del liberalismo y de la ilustración. Gradualmente, la primera rama cobra más importancia, ya que el autor abandona a finales de los años ochenta (porque considera superada) su discusión con NOZICK, RAWLS, DwORKIN o GERIITH.

Whose Justice? Which Rationality? y Three Rival Versions of Moral Enquiry ofrecen más una explicación histórica que una discusión sistemática. MACINTYRE intenta mostrar cómo en un momento de la Ilustración se dio un corte abrupto en la filosofía moral y jurídica: la tradición aristotélica (basada en una antropología naturalista) declina y emerge con fuerza la explicación liberal, de la que RAwLS y DwORKIN son los últimos exponentes.

MACINTYRE historiza la transición desde esta visión aristotélica hacia el liberalismo en la década de 1780 a 1790, en la que los filósofos dejaron de pensar en términos antropológicamente realistas y pasaron hacerlo en términos del sujeto ideal o trascendental ${ }^{24}$. En este diagnóstico coincide parcialmente con FOUCAULT, quien dirigió sus críticas hacia KANT como inventor de un sujeto que jamás había existido. Este es el cambio desde la filosofía naturalista (basada en la biología y la psicología de ARISTÓTELES) a la filosofía transcendental. KANT representa, en este sentido un aliado para el liberalismo, ya que su filosofía es también formal.

A partir de finales del XVIII dejaron de existir razones para actuar, ya que desaparecieron las bases psicológicas y biológicas que proporcionaban una continuidad teleológica entre el conocimiento y la acción. La idea socrática (y aristotélica) del ser inclinado naturalmente hacia el conocimiento y luego hacia la práctica virtuosa quedaba interrumpida. A partir de KANT y de la tradición liberal, el sujeto transcendental era una construcción en la que las razones para actuar no tenían base biológica, sino meramente deontológica.

\section{DESDE WITTGENSTEIN A HART}

Para MACINTYRE, cada sociedad tiene sus propias reglas y prácticas y, al final, todas ellas están limitadas por las inclinaciones naturales de los seres humanos. Todas tienen una convergencia entre las costumbres y los usos de cada lugar y las necesidades de la persona en tanto que «animal racional dependiente» ${ }^{25}$. Esta convergencia, que intenta unir a ARISTÓteles con WitTgENSTEIN, es la base de su pensamiento moral

23 Ibid., 108-199.

24 A. MacInTyre, «How Moral Agents Became Ghosts: Or Why the History of Ethics Diverged from that of the Philosophy of Mind», Synthese, 53, 1982, 295. Vid. también Whose Justice? Which Rationality?, op. cit., 327-335.

${ }_{25}$ Vid. A. MacIntyre, Dependent Rational Animals, Why Human Beings Need the Virtues, Chicago, Open Court, 1999. 
y jurídico. MACINTYRE inicia una teoría natural-consuetudinaria del derecho, que se defiende básicamente en su trabajo «Theories of Natural Law in the Culture of Advanced Modernity» ${ }^{26}$ y en Dependent Rational Animals. El trabajo parte de HART y llega hasta los autores más declaradamente iusnaturalistas. La principal idea es encontrar un «contenido mínimo del derecho natural» a partir de las reglas en las prácticas de cada lugar, y sus costumbres en el marco de las inclinaciones naturales ${ }^{27}$.

Se puede defender que el punto de inicio de la filosofía jurídica de MACINTYRE es la obra de su amigo HART. Tal vez la contribución más relevante de su arranque «iusfilosófico» sea mostrar la contradicción entre, por una parte, la necesidad de un contenido mínimo del derecho natural y, por otra, la reducción del derecho natural a una actividad básica de coordinación. Ciertamente, la admisión de las prácticas y de las reglas sociales de WITTGENSTEIN obligó a HART, positivista convencido, a admitir que entre las reglas sociales había un contenido mínimo de derecho natural, uno de los puntos menos claros de la obra del iusfilósofo de Oxford. En su trabajo «Are There Any Natural Rights?», HART sólo menciona la existencia de un derecho natural: el de la misma aspiración a la libertad de todas las personas ${ }^{28}$, una idea que luego no desarrolla en The Concept of Law. MACINTYRE no explota al máximo esta omisión de HART, sino que defiende que el derecho natural se manifiesta en las inclinaciones y en las costumbres.

Las costumbres no son universales, mientras que las inclinaciones son comunes a todos. Por esa razón, al igual que la libertad vindicada por HART, hay otras tendencias e inclinaciones que tienen los humanos y que luchan para obtener. Ahí se encuentra la base de los derechos naturales. En el artículo «Are There Any Natural Rights?» de MACINTYRE, titulado — no por casualidad - igual que el de HART, MACINTYRE defiende que la prohibición de la esclavitud tiene que ser de derecho natural, por el igual derecho de todos a la libertad ${ }^{29}$.

En este sentido, MACINTYRE muestra cómo una teoría como la de HART debería exigir un contenido más amplio del derecho natural y que, si éste sigue las inclinaciones biológicas y psicológicas del ser humano, puede servir para recomponer las relaciones entre moral y derecho. Del mismo modo, las nociones de justicia y de derechos pueden adecuarse más a la realidad antropológica si, por un lado, se entienden a priori como una costumbre y, por otro lado, a posteriori, como parte de las inclinaciones de los seres humanos.

\section{CONSTRUYENDO EL DERECHO NATURAL}

La concepción del derecho natural de MACINTYRE está construida formalmente sobre ideas de T. DE AQuinO. A pesar de su apertura a los problemas de la Moder-

${ }^{26}$ En E. B. McLeAn (ed.), Common Truths: New Perspectives on Natural Law, Wilmington, ISI Books, 2000, 91-115.

27 A. MACINTYRE, «Theories of Natural Law in the Culture of Advanced Modernity», op. cit., 100.

28 H. L. A. HART, «Are There Any Natural Rights?», Philosophical Review, 64, 1955, 175.

29 A. MacInTYRe, «Are There Any Natural Rights?» (Charles F. Adams Lecture, 28 de febrero de 1983), President and Trustees, Bowdoin College, Brunswick, Maine, 1983, 4. 
nidad, toma como referencia las obras psicológicas y biológicas de ARISTÓTELES y las revisa a la luz de T. DE AQUINO y de los actuales estudios de biología y antropología. MACINTYRE basa el derecho natural en las inclinaciones biológicas y psicológicas del ser humano, visto como un animal racional y dependiente. Su dependencia le hace aprender de los demás y vivir en consonancia con las reglas y costumbres sociales. MACINTYRE sigue a MARITAIN al considerar que las prácticas que los humanos desarrollan en una comunidad durante sus actividades consuetudinarias son la base de los derechos naturales ${ }^{30}$.

Estas condiciones, de acuerdo con lo expuesto en Dependent Rational Animals, permiten el desarrollo individual en el marco de una comunidad. Este aprendizaje práctico está caracterizado por reglas establecidas por la biología humana, que muestra la vulnerabilidad humana y la necesidad de cooperación con los demás ${ }^{31}$. Estas necesidades biológicas no sólo exigen las prácticas y las virtudes de ARISTÓTELES, sino otras como la generosidad o la misericordia que destaca T. DE AQUINO ${ }^{32}$, completando a ARISTÓTELES ${ }^{33}$.

Para MACINTYRE, el Aquinate y el Estagirita explican mejor que nadie las características y las limitaciones de los humanos, ya que se refieren a las personas de carne y hueso. Entendidos como animales racionales, comprenden sus limitaciones y conciben sus vidas como un conjunto de actividades cooperativas con otros ${ }^{34}$. Para MACINTYRE, las decisiones tomadas a priori, con independencia de la sociedad y de las prácticas sociales (como ocurre en el pensamiento liberal) son una mera abstracción.

Así pues, el derecho natural no sólo es un mero conjunto de relaciones de cooperación (como sostiene HART), sino un grupo de prácticas que hacen los seres humanos en sus vidas diarias, reflejando sus inclinaciones biológicas y psicológicas. La transición desde la ética hacia el derecho natural debe hacerse en base a la antropología. MACINTYRE sigue a ARNHART cuando sostiene que ARISTÓTELES y T. DE AQUINO están más cerca que muchos autores modernos del paradigma darwiniano ${ }^{35}$. El filósofo escocés defiende también que los animales tienen una inteligencia, y que aún no se ha podido determinar la conexión de ésta con la humana.

Asimismo, cree que T. DE AQUINO permite una concepción mucho más amplia de la justicia que el propio ARISTÓTELES, ensanchando el catálogo de virtudes de modo que refleja más adecuadamente las inclinaciones psicológicas y biológicas (por ejemplo, la compasión y la generosidad). La justicia tomista, a diferencia de la liberal, no está vinculada sólo al mérito, sino también al conjunto de virtudes propias de los animales racionales dependientes ${ }^{36}$.

MACINTYRE sigue a J. MARITAIN porque defiende que la práctica de la justicia como derecho natural se forja día a día como una práctica del funcionamiento normal

30 A. MACInTYRE, «Theories of Natural Law in the Culture of Advanced Modernity», op. cit., 108-109.

31 A. MacInTYre, Dependent Rational Animals, 10.

32 T. DE AQuino, Summa Theologica, II-II q. 58, a. 3, co.

33 A. MacIntyre, Dependent Rational Animals, op. cit., 93.

${ }^{34}$ Ibid., 124.

35 L. ARnhart, Darwinian Natural Right: The Biological Ethics of Human Nature, Albany, New York, Sunny Press, 1998, 258-266.

36 A. MacInTYre, Dependent Rational Animals, op. cit., 121-125. 
de la naturaleza humana ${ }^{37}$. Contra las interpretaciones intelectualistas (que entienden el derecho natural como una aprehensión intelectual), MACINTYRE se interesa por el proceso de aprendizaje del derecho natural por parte de las personas corrientes (plain persons ${ }^{38}$. En los años noventa, MACINTYRE se opone diametralmente a las ideas de los autores que quieren abrir el tomismo hacia la Modernidad ${ }^{39}$ (como FINNIS o GRISEZ) ${ }^{40}$ o que tienen también una visión intelectualista (M. S. MOORE) ${ }^{41}$ del derecho natural, capaz de ignorar el proceso de aprendizaje de las personas corrientes.

Para MACINTYRE la estrecha relación entre reglas, virtudes y bienes explica cómo las razones para actuar no están sólo de acuerdo con determinadas reglas formales, sino también con la justicia material. Siguiendo estas reglas en la práctica diaria y con la adquisición de los bienes internos (a saber, los que redundan en también en beneficio de la comunidad) a las mismas, se puede llegar a alcanzar una justicia material. Las personas corrientes han aceptado durante centurias la existencia de esta justicia y del derecho natural, pero si el modelo antropológico no es el de la persona de carne y hueso sino el sujeto trascendental, sus expectativas de justicia se quiebran y éstas sólo pueden ser meramente formales.

Para MACINTYRe, si los seres humanos no reconocen su naturaleza animal y racional, con sus limitaciones, tampoco pueden aceptar la idea de Dios ${ }^{42}$. La tradición liberal moderna enfatiza - y casi diviniza - al hombre, entendiéndole capaz de estar más allá de su propia condición natural. A causa de ello, existen dilemas y desacuerdos morales ${ }^{43}$, porque las personas no actúan de acuerdo con su naturaleza social y a menudo en contra de la naturaleza biológica. La falta de naturalidad en relación con los otros y para aprender las prácticas hace que la gente empiece a violar las reglas de la naturaleza y surgen así los desacuerdos. Y éstos no pueden resolverse sólo a través de un incremento de la legislación o de la litigación ${ }^{44}$. Sin embargo, para MACINTYRE, el derecho natural es la base de las reglas de coexistencia de acuerdo con las inclinaciones de los seres humanos. Este modelo tiene ramificaciones políticas: se trata de una visión completamente opuesta al Estado-Nación liberal ${ }^{45}$.

En definitiva, MACINTYRE entiende que su visión permite abrir la filosofía de ARISTÓTELES y de T. DE AQUiNo hacia el pensamiento actual, criticando al mismo tiempo la interpretación más intelectualista de sus doctrinas. Cree que el derecho natural puede ser

37 A. MAcInTYRE, «Theories of Natural Law in the Culture of Advanced Modernity», op. cit., 108-109.

38 A. MacInTyre, «Plain Persons and Moral Philosophy: Rules, Virtues and Goods», American Catholic Philosophical Quarterly, 66, 1992, 10.

${ }_{39}$ G. GRISEZ, «The First Principle of Practical Reason: A Commentary on the Summa Theologiae, 1-2, Question 94, Article 2», Thomist, 21, 1958, 44-78.

40 A. MACINTYRE, «Theories of Natural Law in the Culture of Advanced Modernity», op. cit., 106.

${ }^{41}$ Ibid., 102-103.

42 A. MacInTYRe, Dependent Rational Animals, op. cit., 111.

43 A. MacInTYre, «Moral Dilemmas» en Ethics and Politics: Selected Essays, Volume 2, Cambridge, Cambridge University Press, 2006, 85-100.

${ }_{44}$ Para una explicación más amplia, vid. R. RAMIS BARCELÓ, «MacIntyre y el liberalismo: la correlación entre la ausencia de moral compartida y la proliferación legislativa», Anales de la Cátedra Francisco Suárez, 45, 2011,333-348.

${ }_{45}$ A. MacIntyre, «Natural Law against the Nation-State: Or the Possibility of the Common Good against the Actuality of the Public Interest», en The 1994 Agnes Curning Lectures in Philosophy delivered at University College, Dublin, on March 1, 2, and 3, 1994. 
captado como un conjunto de reglas que se construyen con la práctica diaria. Siguiendo las reglas en estas prácticas es posible la resolución de los desacuerdos morales.

\section{RECONSIDERANDO EL DERECHO NATURAL}

Contra la supremacía de la política sobre la ética (típica de la época Moderna), MACINTYRE defiende la supremacía de la ética sobre la política. Las comunidades son primeramente un grupo de personas que guían sus prácticas buscando el bien común desde la práctica de las virtudes públicas (que no son sólo la excelencia, sino también la justicia como caridad, misericordia, compasión...) Es lo que nos hace animales y lo que nos hace humanos, tal y como muestra la antropología biológica de los sentimientos.

Sin embargo, tanto ARIsTóteles como T. DE Aquino defendieron la práctica de las virtudes en pequeñas comunidades, no en el marco de los grandes Estados Nacionales, que surgieron después. MACINTYRE defiende que sólo en comunidades cuya extensión sea (como máximo) la de la civitas o la pólis, puede darse un aprendizaje de las prácticas siguiendo las inclinaciones naturales. En el Estado-Nación se disuelven estos hábitos de aprendizaje y se transforman en algo meramente impersonal, formal y abstracto.

Si las comunidades están gobernadas por las inclinaciones naturales y el aprendizaje de las prácticas, esto dará lugar a hábitos y costumbres que tengan en cuenta el bien común. Según T. DE AQUiNo hay unos primeros principios de la ley natural que son inmutables (no robar, matar, mentir...) y otros, secundarios, que son más adaptables a ciertas costumbres del lugar, pese a ser algo extrañas o incluso desviadas ${ }^{46}$. En todo caso, el derecho natural tiene para MACINTYRE una carga subversiva y suele oponerse, más que a la costumbre desviada, al abuso ${ }^{47}$. Por ejemplo, B. DE LAS CASAS defendió, en base a los primeros principios del derecho natural, que las costumbres «desviadas» de los indios no permitían actuar contra ellos ${ }^{48}$.

MACINTYRE entiende que las personas corrientes pueden familiarizarse con el derecho natural al buscar los bienes internos en cada una de las prácticas diarias, aunque parezcan extrañas a las que no están acostumbradas a ellas. La idea de fondo es que las personas corrientes no pueden captar el derecho natural y no pueden resolver problemas en los se exija una operación de abstracción: sólo pueden aprender a través de las prácticas sociales y de las costumbres.

Esta visión antiintelectualista implica, como se verá, graves problemas en el seno de una filosofía tomista. MACINTYRE, pese a mantener una actitud antimoderna, procura demostrar en los últimos años la superioridad del tomismo a través de una serie de acuerdos puntuales con la obra de KANT ${ }^{49}$, principalmente, pero también con la de

46 A. MacIntyre, God, Philosophy, Universities. A Selective History of Catholic Tradition, Maryland, Rowman \& Littlefield Publishers, 2009, 89.

47 A. MacInTyRe, «Natural Law as Subversive: The Case of Aquinas», en Ethics and Politics, Selected Essays, op. cit., 41-63.

48 A. MACInTyre, God, Philosophy, Universities. A Selective History of Catholic Tradition, op. cit., 108.

49 A. MAcInTyre, «Truthfulness and Lies: What We Can Learn from Kant», en Ethics and Politics, Selected Essays, op. cit., 122-142. 
Mill. Aunque MacInTYRE adopta una actitud anti-moderna en toda su obra, en sus últimos trabajos, ya en la primera década del siglo XXI, intenta una convergencia con la ley moral en KANT.

Para el filósofo escocés puede existir un descubrimiento de los principios del derecho natural en el diálogo racional, un extremo que le acerca a la deliberación kantiana y, sobre todo, neokantiana. La diferencia es que MACINTYRE cree posible dar una respuesta material a los desacuerdos morales, mientras que los pensadores inspirados por KANT dan sólo una solución formal ${ }^{50}$. Para MACINTYRE, si una comunidad toma a todos sus miembros sin exclusiones, con iguales condiciones, con el compromiso de decir la verdad, abandonando el uso de la violencia y respetando la racionalidad para resolver los desacuerdos morales, para él estas reglas no son sino los primeros principios del derecho natural. Si la gente sigue en sus prácticas dichas reglas, los desacuerdos morales pueden desaparecer ${ }^{51}$.

La idea de que las personas corrientes pueden aprender los principios del derecho natural a partir de la búsqueda de los bienes internos a las prácticas es problemática. MACINTYRE cree que la gente puede hallar el derecho natural sin ninguna captación intelectual. Es posible pensar que las personas corrientes no son capaces de un acto de aprehensión, pero al mismo tiempo MACINTYRE entiende que son hábiles para manejarse en un debate racional y que pueden hallar los principios de derecho natural si ambos siguen las prácticas de debate antes apuntadas.

El filósofo escocés quiere mostrar que su teoría del derecho natural, a partir de las prácticas, es capaz de superar el utilitarismo y su doctrina del consecuencialismo moral. Algunos de los debates consecuencialistas son irresolubles y, según el filósofo escocés, sólo los preceptos del derecho natural pueden resolver el problema. Sin embargo, ¿aparecen dichos preceptos sólo en el contexto del desacuerdo moral?, ¿cómo se puede saber si un problema ha quedado definitivamente resuelto?

MACINTYRE articula una síntesis de la ética aristotélico-tomista y de la teoría wittgensteiniana de la acción ${ }^{52}$. Para ANSCOMBE, la filosofía práctica explica que hay razones objetivas para la acción. Si en los escritos de juventud, MACINTYRE se pregunta acerca de la falta de razones para la acción, en sus obras tardías, es capaz de resolver esta cuestión integrando el primer principio de la razón práctica de T. DE AQUINO (hacer el bien y evitar el mal) con las prácticas de WiTTGENSTEIN ${ }^{53}$.

Así, el razonamiento práctico, basado en hacer el bien y evitar el mal significa, para MACINTYRE, la primera razón para actuar. Para él, los seres humanos necesitan buscar los bienes internos a cada práctica como una razón para actuar. Las razones para la acción dependen de las prácticas de algunos bienes hacia los que los seres humanos están inclinados. El primer principio de la razón práctica se dirige hacia la efectiva realización del bien gracias al conocimiento y a la práctica de los primeros principios del derecho natural. Las acciones, como indica ANSCOMBE, deben ser evaluadas en el

50 A. MacIntyre, God, Philosophy, Universities. A Selective History of Catholic Tradition, op. cit., 91.

51 A. MacInTyre, «Aquinas and the Extent of Moral Disagreement», en Ethics and Politics, Selected Essays, op. cit., 64-82.

52 E. PerREAu-Saussine, Alasdair MacIntyre: Une biographie intellectuelle, Paris, PUF, 2005, 88.

53 A. MacIntyre, God, Philosophy, Universities. A Selective History of Catholic Tradition, op. cit., 90. 
contexto en el que los agentes narran sus propias intenciones. En el ejercicio de las prácticas, los agentes encuentran las virtudes que ayudan a los seres humanos al florecimiento individual de acuerdo con el bien común ${ }^{54}$.

El hecho de que el derecho natural sea una guía para la acción y que se encuentre en la práctica de acuerdo con las inclinaciones naturales, resuelve para MACINTYRE el problema del aprendizaje de los derechos naturales. De acuerdo con los autores más cognoscitivistas (por ejemplo, GRISEZ, FINNIS o VILLEY), la captación del derecho natural es una operación abstracta. MACINTYRE cree que la gente corriente no es capaz de hacerla. En cambio, cree que la gente puede aprender las reglas del derecho natural en las prácticas racionales y comunitarias. Así, los seres humanos pueden enseñar unos a otros $\mathrm{y}$, en un debate racional, hallar los preceptos del derecho natural ${ }^{55}$.

Los tomistas «clásicos» tienden a defender la primacía de la teología sobre la comprensión del derecho natural, y tienen una actitud anti-moderna, contraria al subjetivismo de KANT y se oponen a la idea de los derechos humanos ${ }^{56}$. Los tomistas «neoclásicos» entienden que el derecho natural es aprehendido por la razón, es independiente de la teología, su actitud es abierta hacia la Modernidad, a los derechos humanos (como secularización del derecho natural) y aceptan la existencia de la falacia naturalista (entendida como la prohibición de derivar imperativos prácticos de premisas obtenidas de la razón teorética) y buscan una conexión entre KANT y T. DE AQUiNO.

MACINTYRE comparte con los «tomistas clásicos» la anti-modernidad y el rechazo de los derechos humanos. Sin embargo, como hacen los «neoclásicos» ${ }^{57}$, mantiene la independencia de la filosofía frente a la teología y últimamente busca una aproximación, desde el tomismo, a KANT y a MiLL para mostrar que tienen algunas ideas que pueden recuperarse. Pese a su condición de filósofo tomista, considera que el estudio del derecho natural es una cuestión en la que la filosofía no necesita la injerencia de la teología. Ésta viene a corroborar y a certificar lo que la filosofía puede entender por sí misma. A diferencia de J. PORTER y de otros teólogos, MACINTYRE vindica la independencia de la filosofía para conocer el derecho natural ${ }^{58}$.

En definitiva, la obra de MACINTYRE, ante todo, es una contribución significante en tres áreas: la primera, una explicación de cómo se puede aprehender el derecho natural; la segunda, un examen de cómo el derecho natural entendido como práctica puede tener un papel preeminente para resolver desacuerdos morales y, finalmente una presentación de la actual proliferación legislativa como una ausencia de virtudes éticas y una falta de compromiso con la práctica del derecho natural.

54 Ibid., 162.

55 A. MaCInTYRE, «Intractable Moral Disagreements», en L. S. Cunningham (ed.), Intractable Disputes About the Natural Law. Alasdair MacIntyre and Critics, op. cit., 4-8.

56 A. MacInTyre, «Community, Law, and the Idiom and Rhetoric of Rights», Listening: Journal of Religion and Culture, 26, 1991, 96.

57 A. Lisska, Aquinas's Theory of Natural Law: An Analytic Reconstruction, Oxford, Clarendon Press, $1996,2-5$.

58 A. MaCInTYRE, «From Answers to Questions», en L. S. CunNINGHAM (ed.), Intractable Disputes About the Natural Law. Alasdair MacIntyre and Critics, op. cit., 315. 


\section{BALANCE Y CRÍTICA: EL DESARROLLO DE VARIAS LÍNEAS}

A mi entender, MACINTYRE tiene dos líneas intelectuales en su visión del derecho. Una es la conexión ARISTÓtELES-MARITAIN-WitTGENSTEIN-HART y la otra es la línea Sócrates-Aristóteles-T. De Aquino-Kant-Anscombe-Maritain. Dependent Rational Animals es una expresión de la primera línea, mientras que «Intractable Moral Disagreements» muestra la convergencia con la segunda. Son dos versiones ad extra del tomismo de MACINTYRE, expuestas para un diálogo con los autores de otras tradiciones. Al mismo tiempo, el autor tiene también una visión ad intra, expresada en su libro God, Philosophy, Universities ${ }^{59}$.

Cabe preguntarse si las visiones anteriores son compatibles entre sí. Creo que sólo parcialmente. Por una parte, la antropología de la persona de carne y hueso es compatible con una antropología que llega hasta Hume pero no con la concepción antropológica y con la ley moral de KANT. El racionalismo de T. DE AQUINO no es compatible con la concepción del segundo WiTTGENSTEIN, aunque sí lo es con la ley moral en KANT. El tomismo requiere que las decisiones deben ser racionales de acuerdo con la antropología de la persona, en las que hay una pasión controlada por la razón. MACINTYRE parece sugerir una racionalidad basada en las reglas sociales y un vínculo con la pasión y con la racionalidad de los animales, aunque por otro lado parece vindicar el racionalismo clásico de la visión tomista.

Se plantean dos cuestiones: ¿cuál es el papel de los sentimientos en el aprendizaje? Y, ¿por qué deben existir las mismas reglas para todos? Siguiendo a WITTGENSTEIN, MACINTYRE debería admitir que las reglas tienen que ser diferentes en cada contexto social, pero de acuerdo con el universalismo racional que exige el tomismo (y KANT), parece que las reglas de cada comunidad no son válidas. Para T. DE AQUINO hay unos primeros preceptos de la ley natural que están vinculados con el primer principio de la razón práctica (hacer el bien y evitar el mal) y otros, que son secundarios, que tienen un carácter más mutable ${ }^{60}$.

MACINTYRE parece invertir el esquema de T. DE Aquino. De entrada, hay una serie de reglas y de prácticas consuetudinarias en cada comunidad, que pueden ser más o menos racionales. Sin embargo, el escocés cree que todas ellas, si buscan los bienes internos a las prácticas tienden a encontrar los «principios del derecho natural» que, en última instancia, son parecidos para todos. Las personas, pese a que en diferentes lugares utilicen prácticas y reglas diferentes, si buscan los bienes internos a las prácticas pueden hallar los preceptos del derecho natural.

Esta visión tiene varios problemas. En primer lugar, no es compatible para un tomista determinar un conocimiento directo de los preceptos de la ley natural que se pueden conocer por analogía y sostener, como hace MACINTYRE, una visión sociologista e inductiva (en la que cree que, a pesar de las muchas diferencias que hay en las prácticas y en las reglas de cada sociedad, si todas ellas siguen los bienes internos, hallarán los preceptos del derecho natural).

\footnotetext{
59 A. MacIntyre, God, Philosophy, Universities, op. cit., cap. 10.

60 T. DE AQuino, Summa Theologica, I. ${ }^{\text {a }}$-IIae q. 94 a. 6 co.
} 
En segundo lugar puede destacarse una dificultad epistemológica. Si el derecho natural se descubre en la práctica y si la resolución de los conflictos se tiene que hacer en base al debate racional, ¿puede una persona corriente encontrar el derecho natural y alcanzar una solución usando un proceso racional? y ¿hasta qué punto se exige racionalidad en las prácticas y no captación intelectual? Esta visión pretende seguir a WitTGENSTEIN, pero se parece mucho más a la mayéutica socrática, que anuda el aprendizaje progresivo con la aproximación gradual a la verdad ${ }^{61}$. Y, desde luego, la senda convencionalista de WITTGENSTEIN no se refiere a la aproximación a la verdad en las prácticas, mientras que la línea tomista (y también KANT) buscan la «verdad».

En tercer lugar, ¿es compatible una aproximación convencional-consuetudinaria a partir de las reglas y de las prácticas con un acto de captación intelectual por analogía? ¿Se puede llegar a un conocimiento del derecho natural sin que, a la postre, haya una firme convicción de que el proceso inductivo desemboca en la verdad? Parece que MACINTYRE exige acomodar muchas ideas diferentes en su misma doctrina. Para solventar estas dificultades, se plantean varias opciones:

a) Si tomamos las prácticas sociales como punto de partida, se puede argumentar que hay muchas inclinaciones naturales de las personas y que pueden buscar los bienes internos a las prácticas, aunque éstas no estén subordinadas a la verdad. Sería una «razón práctica», independiente de la teorética, basada en un naturalismo no intelectualista. En este caso, no se buscaría un bien universal, sino uno basado en las reglas consuetudinarias de cada sociedad, fundamentado en las inclinaciones biológicas, psicológicas y sociales de los seres humanos y en su búsqueda comunitaria de los bienes internos a sus prácticas.

Sin embargo, considérese, por ejemplo, la eugenesia positiva de un grupo que busca seleccionar la raza. Podría ser considerada una práctica natural (hay sociedades tribales que lo hacen con «naturalidad») porque estas personas tienen anormalidades que impiden su participación plena en actividades de grupo. Las personas deformadas pueden contribuir muy poco al grupo, pero en cambio consumen sus recursos. Esta podría ser una razón (consecuencialista) para la práctica de estas personas, que MACINTYRE rechazaría de plano.

b) Si tomamos como referencia las inclinaciones naturales como un bien para la persona humana, éstas pueden tener un valor antropológico para su vida personal o comunitaria, pero no es necesariamente universal. Siguiendo las inclinaciones naturales (biológicas o psicológicas) o las prácticas consuetudinarias, una persona puede no obtener necesariamente ningún tipo de verdad, pero ello sirve para mostrar una tendencia en la adaptación de la acción humana a las prácticas. Seguir las inclinaciones humanas no garantiza el acceso a la verdad. En todo caso, éstas podrían ser consideradas un conjunto de costumbres basadas en la naturaleza biológica de los seres humanos.

Considérese, por ejemplo, el caso de la guerra, que es claramente un instinto animal y no necesariamente una práctica universal. Sostener que la guerra puede ser un bien para una comunidad es sólo una costumbre (o una convención) humana, de acuerdo

${ }^{61}$ Vid. T. IRwIN, The Development of Ethics, vol. I, Oxford, OUP, 2007, capítulo 2. 
con el instinto animal y biológico que tienen los humanos. Otro ejemplo podría ser la caza, otro instinto animal, parte de los hábitos de muchas comunidades. La práctica de la caza puede ser entendida como un bien para una comunidad que busca su supervivencia y su alimentación, mientras que para otras podría entenderse como un acto contrario a la naturaleza.

c) Si el bien puede ser conocido y es consistente con la verdad (unidad de la razón teórica y la razón práctica), las prácticas no son convencionales o consuetudinarias, sino que ellas deben estar subordinadas al conocimiento del bien y que este bien sólo puede encontrarse en la verdad. En este sentido podría decirse que hay varias prácticas que pueden corregirse al invocar un concepto de bien consistente con la verdad, o las prácticas pueden dirigirse simultáneamente a varios «bienes» y «verdades» incompatibles entre sí (es decir, que no haya ni un único summum bonum ni una sola verdad).

d) Sin embargo, MacInTYRE, pese a proclamar su tomismo, no acaba de defender completamente ninguna de las tesis anteriores. Su punto de partida es la persona corriente que no puede captar ni el primer principio de la razón práctica ni los preceptos del derecho natural. En cambio, pueden «aprenderse» en prácticas convencionales y consuetudinarias que las personas reciben en sus comunidades a través de las enseñanzas que reciben de las otras. Cada una de esas prácticas no viola la naturaleza biológica y psicológica del ser humano, pero las prácticas deben basarse en sus inclinaciones y en la búsqueda del bien interno.

Como defiende MARITAIN, dicha búsqueda es una actividad racional que lleva a cabo toda persona cuando está «funcionando» normalmente. Este aprendizaje dialéctico confirma que las reglas de la justicia y del derecho natural contienen un valor de verdad porque la persona experimenta una progresión y un enriquecimiento que gradualmente confirma el valor de cada práctica como verdad. Sin embargo, el fundamento de MARITAIN dista de ser meramente consuetudinario y de estar basado en las reglas sociales. Para él, el derecho natural se basa en la libertad y en la dignidad especial del sujeto, extremo que MACINTYRE no desarrolla específicamente.

En cambio, el escocés parece un autor enteramente socrático, en su aproximación a la verdad, pero contrario a la captación intelectualista del derecho natural. El modelo antropológico sobre el que MARITAIN construye su derecho natural es el ser humano entendido como una criatura racional y dotada de una especial dignidad, que le lleva hacia la defensa de la libertad como bien sobre el que articula su ética y que el derecho natural defiende. Para MACINTYRE, el ser humano es un «animal racional dependiente» caracterizado por su fragilidad, que va encontrando el derecho natural en sus prácticas diarias, guiado por la comunidad en la que todas las reglas y costumbres adquieren sentido. La visión de MACINTYRE parece muy optimista, ya que su hipótesis parece sostener que las prácticas y las costumbres de los grupos sociales, al final, acaban coincidiendo con las deliberaciones de los seres humanos que siguen sus inclinaciones naturales.

Precisamente, este argumento d) presenta algunos puntos muy cuestionables. Primero, el hecho de decir que las prácticas sociales no van en contra de la naturaleza de las personas es una hipótesis muy dudosa y tiene muchos ejemplos en contra. El ejem- 
plo propuesto por MACINTYRE sobre la esclavitud es problemático. Para la persona de hoy, la dignidad humana prohíbe directamente la esclavitud y pero muchos filósofos de Grecia y Roma, que no eran precisamente personas corrientes, no entendieron que la esclavitud conculcara ningún derecho natural.

Esta idea muestra que el derecho natural es poco estático sino más bien un concepto cambiante, de acuerdo a los usos, costumbres y convenciones de los seres humanos. Hay muy pocos casos en los que casi todas las sociedades de diferentes épocas estén convencidas de la existencia de un derecho natural. Por lo tanto, la presunción de MACINTYRE de que el derecho natural se puede encontrar en las prácticas desemboca en la existencia de tantos derechos naturales como prácticas, algo que es contrario a la verdad, a menos que se acepte la existencia de varias verdades y de varios «derechos naturales», extremo que el filósofo escocés desaprueba.

Segundo, del mismo modo, la aceptación de la idea de MARITAIN, en la que el derecho natural es una suerte de sindéresis o de habilidad para funcionar de acuerdo con el sentido común ${ }^{62}$ evita, ciertamente, la idea de la captación intelectual, pero atenúa mucho captación analógica del bien exigida por T. DE AQUINO, así como la necesidad de la existencia de la verdad como guía para la actuación. Para MACINTYRE, el contraste de una práctica con la verdad puede examinarse si ésta rompe dramáticamente con las costumbres y prácticas establecidas ${ }^{63}$. Esta hipótesis parece poco plausible, ya que se han suprimido drásticamente muchas costumbres, que hoy parecen contrarias al derecho natural, precisamente por el hecho de contravenir el statu quo (piénsese, por ejemplo, en las revoluciones del siglo XVIII).

Esta idea de hallar del derecho natural en el aprendizaje de las prácticas tiene mucho interés, pero, para articularla, MACINTYRE intenta — a mi entender- conjugar demasiadas líneas, que resultan problemáticas y muy difíciles de armonizar. Para unirlas, debe recurrir a Dios, un elemento filosóficamente muy problemático. A través de un Dios (más tomista que aristotélico) que atrae a los entes hacia sí, MACINTYRE encuentra un garante de que todas en las prácticas — por diferentes que sean — si se persigue el bien interno, se pueden hallar principios de derecho natural.

Así, Dios es la garantía de la verdad que exigen en su concepción del bien tanto Sócrates como T. DE Aquino o KANT ${ }^{64}$. Por ese motivo, MacInTyRe accede a Dios para garantizar que toda práctica que busque el bien interno hallará los principios de derecho natural puestos por Dios, ya que Él es el summum bonum y el garante de la verdad ${ }^{65}$. Evidentemente, esta teodicea, establecida por mor de la verdad y como sostén de un edificio intelectual problemático, plantea de nuevo numerosas dificultades que el lector comprende de entrada y sin necesidad de abundar mucho más en la cuestión.

${ }^{62}$ J. Maritain, Natural Law: Reflections on Theory and Practice, South Bend, St. Augustine Press, $1943,20$.

${ }_{63}$ A. MacIntyre, «Narrative Ethics, Virtue Ethics and Natural Law» (Conferencia), septiembre $1990,2$.

${ }^{64}$ A. MacIntyre, «Truthfulness and Lies: What We Can Learn from Kant», en Ethics and Politics, Selected Essays, op. cit., 124-131.

${ }_{65}$ A. MacInTyre, «How Can We Learn What Veritatis Splendor Has to Teach?», Thomist, 58 (1994), 173. 


\section{CONCLUSIONES}

En los años cincuenta y sesenta, MACINTYRE se interesa en la falta de las razones para actuar y en la brecha entre el conocimiento y la decisión. Para estudiarla, acude a la filosofía griega. Para él el liberalismo y la Ilustración representan la contravención de la antropología natural defendida hasta KANT. A partir de KANT, la antropología basada en los rasgos psicológicos y biológicos del ser humano desapareció y fue sustituida por una epistemología trascendental, basada en el sujeto trascendental.

Asimismo, mientras el derecho natural en la tradición aristotélico-tomista era visto como un producto de la justicia y de otras virtudes éticas, el liberalismo divorció el derecho de la ética, haciendo una lectura política. Cuando la ética naturalista y el derecho natural desaparecieron, empezaron a aparecer insalvables desacuerdos morales y jurídicos que el liberalismo no podía resolver. Esta sería la reconstrucción macintyreiana de la caída del derecho natural y el estado del derecho en la actualidad.

La falta de razones para actuar viene dada porque actualmente hay una antropología basada en el sujeto trascendental, que descuida las inclinaciones biológicas y psicológicas del ser humano. Al deslindar la razón teórica de la razón práctica, los imperativos de la razón práctica y las prácticas quedan sin un determinado contexto. Cuando desaparece la ética basada la biología y en la antropología, se abandona el aprendizaje natural de la virtud y el bien. De este modo se sustituye por una proliferación legislativa y se producen desacuerdos y dilemas morales que tienen consecuencias jurídicas.

Para superarlo, MACINTYRE propone una teoría del derecho natural basada en las inclinaciones antropológicas del ser humano. El filósofo escocés está en deuda con WiTTGENSTEIN, ANSCOMBE y HART, porque en ellos encuentra el camino para articular una teoría no intelectualista del derecho natural. Al mismo tiempo, como aristotélico y tomista, tiene que aceptar toda una serie de exigencias, como la de la equivalencia entre el bien y la verdad, y la de su captación racional.

Su propuesta tiene una tensión interna entre una comprensión basada en las prácticas y las reglas (de carácter consuetudinario) y otra basada en valores universales. Esta postura puede entenderse como una reformulación del pensamiento de HART para combinarlo con algunas exigencias aristotélicas o tomistas (cercanas a MARITAIN). Si la verdad no es una exigencia, puede haber un camino para una teoría naturalconsuetudinaria, que une a ARISTÓTELES con WITTGENSTEIN.

MACINTYRE, con todo, es un tomista, y la unidad del bien y de la verdad es una exigencia universal para el estudio del derecho natural. En Intractable Disputes about the Natural Law, MACINTYRE explica que el derecho natural aparece en la resolución de los desacuerdos morales si las personas persiguen los bienes internos a las prácticas y adoptan una actitud de debate racional en la que los contertulios busquen la verdad.

La argumentación de MACINTYRE tiene algunos problemas, entre ellos, que la deliberación es una actividad que no puede ser llevada a cabo al máximo nivel por las personas corrientes y que la visión que aúna a ARISTÓTELES, WITTGENSTEIN y HART no es compatible con la que integra a Sócrates y T. DE AQUINO, y que asimismo busca algunos puntos de contacto con KANT. 
Me resulta difícil dar alguna respuesta a este problema y me confieso incapaz de hacerlo. Sólo puesto en la tesitura de buscar obligatoriamente alguna solución, creo que tal vez MACINTYRE debería decidirse por alguna de las líneas que he detectado en sus escritos de las últimas décadas y que he expuesto antes. Si aboga por un modelo tomista, debería recortar algunas concesiones a la teoría de WITTGENSTEIN y sería casi incompatible con algunas ideas de HART; mientras que si se decide finalmente por una visión basada en el aprendizaje en las prácticas y en las costumbres, tiene que rechazar algunos elementos teológicos o metafísicos. No veo que el «sistema» pueda seguir funcionando sin renunciar a una de estas dos grandes líneas.

Desde luego, el modelo consuetudinario es más novedoso y le permitiría integrar a WiTTGENSTEIN y MARITAIN, en una opción biologicista, sociológica y antropológica, que podría explorar a fondo el «contenido mínimo del derecho natural» de HART entendido como un derecho de la persona a la libertad, que MARITAIN pone como base de su concepción filosófica. En todo caso, es una opción intelectual muy personal que tal vez no acabe de perfilarse nunca con claridad.

En definitiva, MACINTYRE es un importante teorizador e historiador de los problemas éticos de nuestra sociedad, y su explicación de los actuales problemas legislativos y de los desacuerdos morales tiene un gran atractivo. Su doctrina del derecho natural tiene muchas sugerencias e interesantes críticas, aunque - como se ha pretendido mostrar en este escrito- hay algunas cuestiones que quedan sin fundamentar o dificultades que permanecen sin resolver. Con todo, MACINTYRE puede considerarse uno de los pensadores que ha terciado con mayor originalidad en el debate iusfilosófico de nuestros días. 